\title{
A formalization of one of the main claims of "The cancer glycocalyx mechanically primes integrin-mediated growth and survival" by Paszek et al. $2014^{1}$
}

\author{
Russell Bainer \\ Maze Therapeutics, USA \\ E-mail: rbainer@mazetx.com; ORCID: https://orcid.org/0000-0002-0830-7029 \\ Editor: Cristina-Iulia Bucur (https://orcid.org/0000-0002-7114-6459) \\ Review comments from: Tobias Kuhn (https://orcid.org/0000-0002-1267-0234); Michel Dumontier \\ (https://orcid.org/0000-0003-4727-9435); Margherita Martorana (https://orcid.org/0000-0001-8004-0464); Cristina-Iulia \\ Bucur (https://orcid.org/0000-0002-7114-6459)
}

Received 18 June 2021

Accepted 29 November 2021

Abstract. Paszek et al. claimed in previous work that glycocalyx bulk mechanically drives integrin clustering in cancer cells engaged with the extracellular matrix. We present here a formalization of that claim, stating that all things of class "glycocalyx bulk" that are in the context of a thing of class "ecm bound cancer cell" generally have a relation of type "increases" to a thing of class "integrin clustering" in the same context.

Keywords: ecm bound cancer cell, glycocalyx bulk, integrin clustering

\section{Introduction}

Paszek et al. [2] state that "Expression of large tumour-associated glycoproteins in non-transformed mammary cells promoted focal adhesion assembly and facilitated integrin-dependent growth factor signalling to support cell growth and survival.’. We present here a formalization of the main scientific claim from this quote by using a semantic template called the super-pattern [1].

\section{Formalization}

Our formalization looks as follows:

\footnotetext{
${ }^{1}$ As RDF/nanopublication: http://purl.org/np/RAoo8EvTgfkxJw5SgZXbJvR15nQG7ygeGaHp8Zud1U4Zw
} 


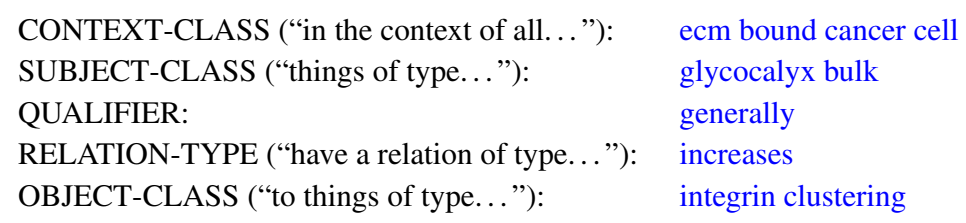

In the context class we use a new minted class "ecm bound cancer cell" that is a subclass of "cancer cell" (Q4118072) from Wikidata and is related to the class "extracellular matrix" (Q193825) from Wikidata. In the subject class, we use a new minted class "glycocalyx bulk" that is related to the class "glycocalyx" (Q898356) from Wikidata. In the object class we minted a new class "integrin clustering" that is a subclass of "integrin binding" (Q14633861) from Wikidata and is related to the class "focal adhesion" (Q904514) from Wikidata.

\section{RDF code}

This is our formalization as a nanopublication in TriG format:

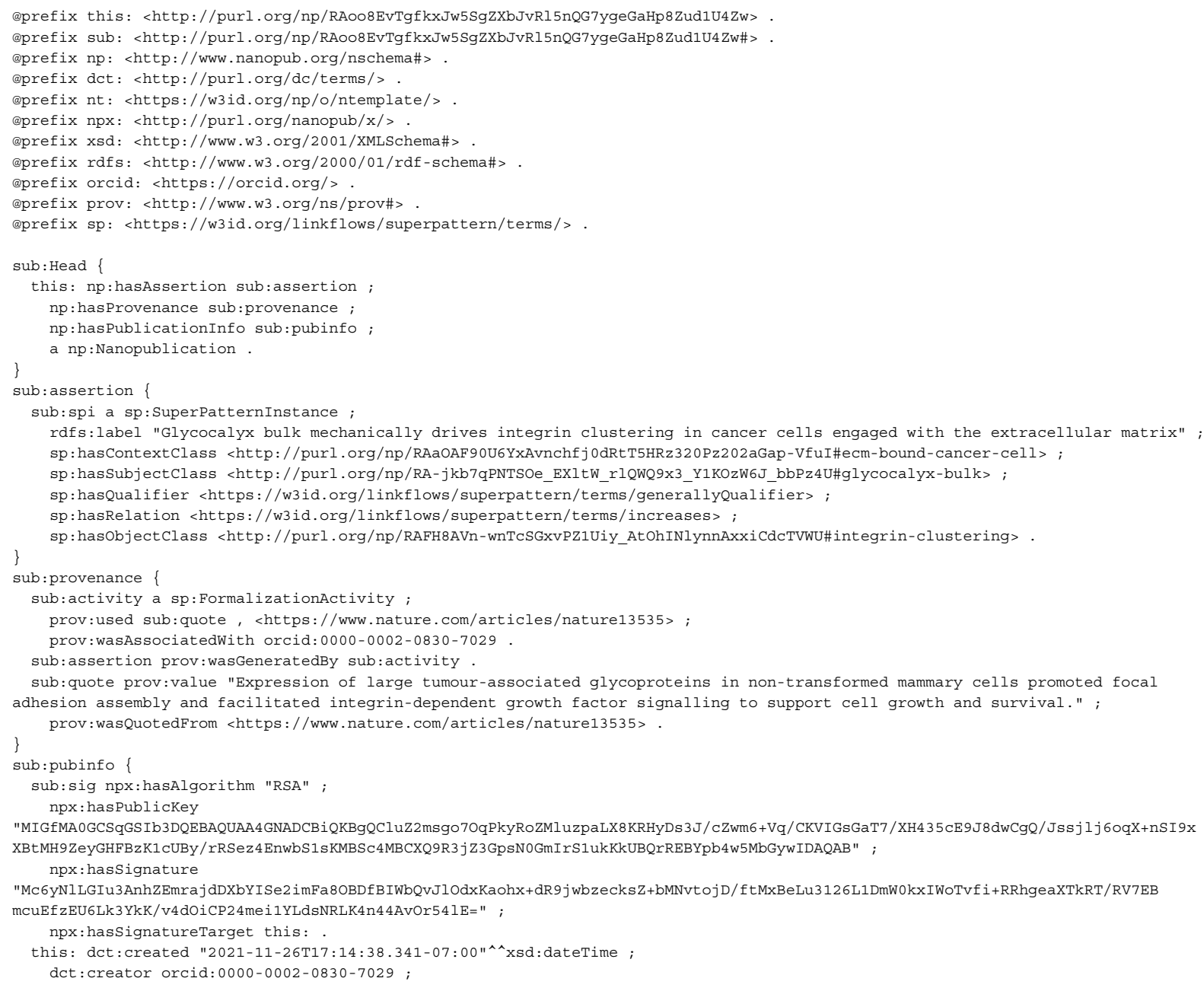


npx: introduces sub:spi ;

npx: supersedes <http://purl.org/np/RAh1GOK4_HhqeUeJhIYV_DDCFFTY9jeeiKWgbSzooWEi4>

<https://w3id.org/linkflows/reviews/isUpdateof> <http://purl.org/np/RASZZ5T1Ca5gpCMPubKoypR_0WcSUkHiMaADu9o1BS1Xs> ; $\mathrm{nt}$ : wasCreatedFromProvenanceTemplate <http://purl.org/np/RAE1wniOy0yO39PlK9QkQ-wqbC3q-R2nXraP5huu8W39k> ;

nt: wasCreatedFromPubinfoTemplate <http://purl.org/np/RA2vCBXZf-icECVRGhulJXugTGxpsV5yVr9yqCI1boh4A>

<http://purl .org/np/RAA2MfqdBCzmz9yVWjKLXNbyfBNCwSMmOqCNUxkk1maIM>

<http://purl .org/np/RAjpBMlw3OwYhJUBo3DtsuDlXsNAJ8cnGeWAutDVjuAuI> ;

nt: wasCreatedFromTemplate <http://purl.org/np/RAv68imZrEjfcp2rnEg1hzoBqEVc0cQMtp9_1Za0BxNM4 >

The following nanopublications introduce the newly minted classes in TriG format.

This is the class definition of "ecm bound cancer cell":

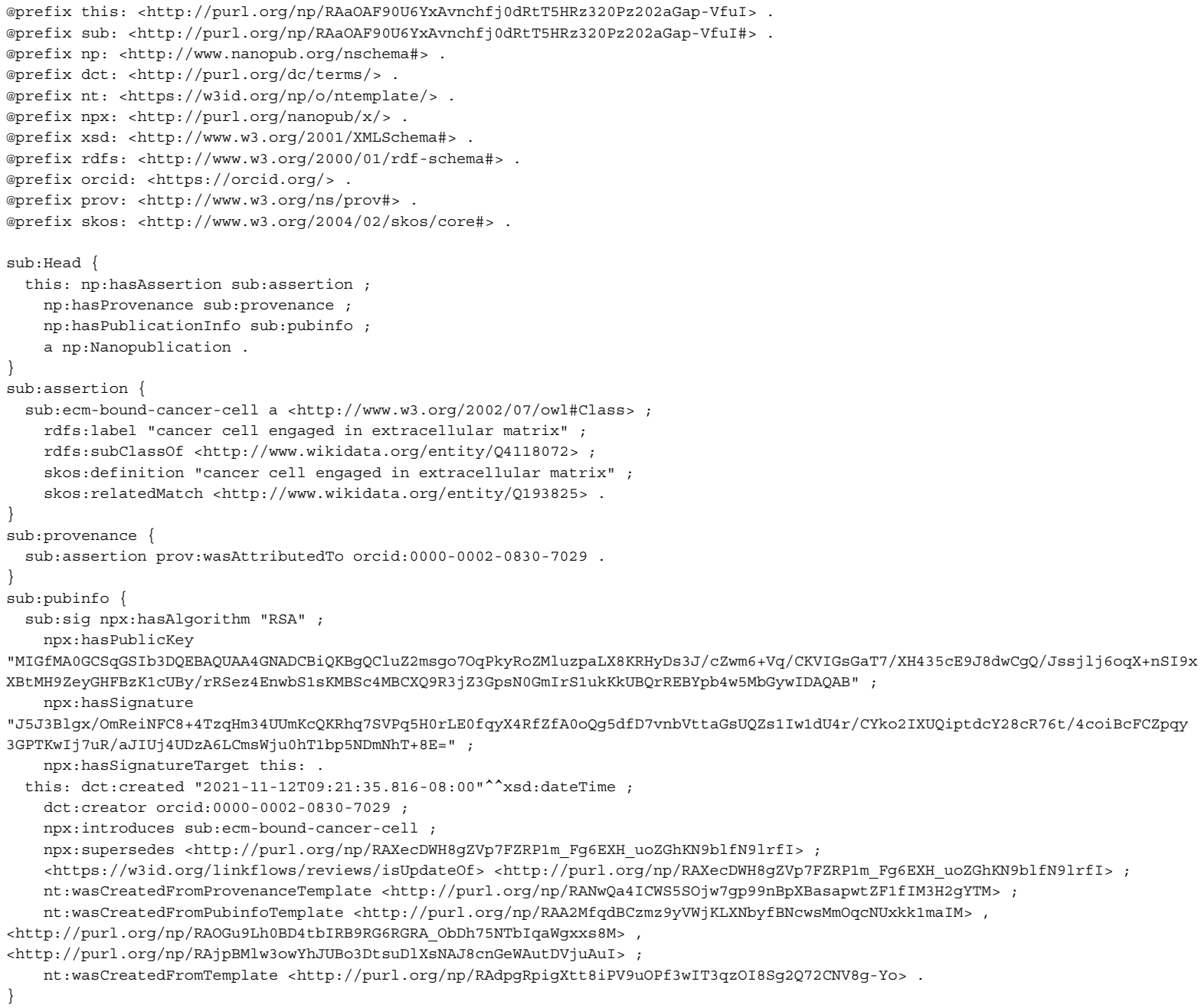

This is the class definition of "glycocalyx bulk":

@prefix this: <http://purl.org/np/RA-jkb7qPNTSOe EXltW rlQWQ9x3 Y1KOzW6J bbPz4U> . @prefix sub: <http://purl.org/np/RA-jkb7qPNTSOe_EXltW_rlQWQ9x3_Y1KOzW6J_bbPz4U\#> @prefix np: <http://www.nanopub.org/nschema\#> . $@$ @refix dct: <http://purl.org/dc/terms/>

@prefix nt: <https://w3id.org/np/o/ntemplate/>

@prefix npx: <http://purl.org/nanopub/x/>

@prefix xsd: <http://www.w3.org/2001/XMLSchema\#>

@prefix rdfs: <http://www.w3.org/2000/01/rdf-schema\#> . 


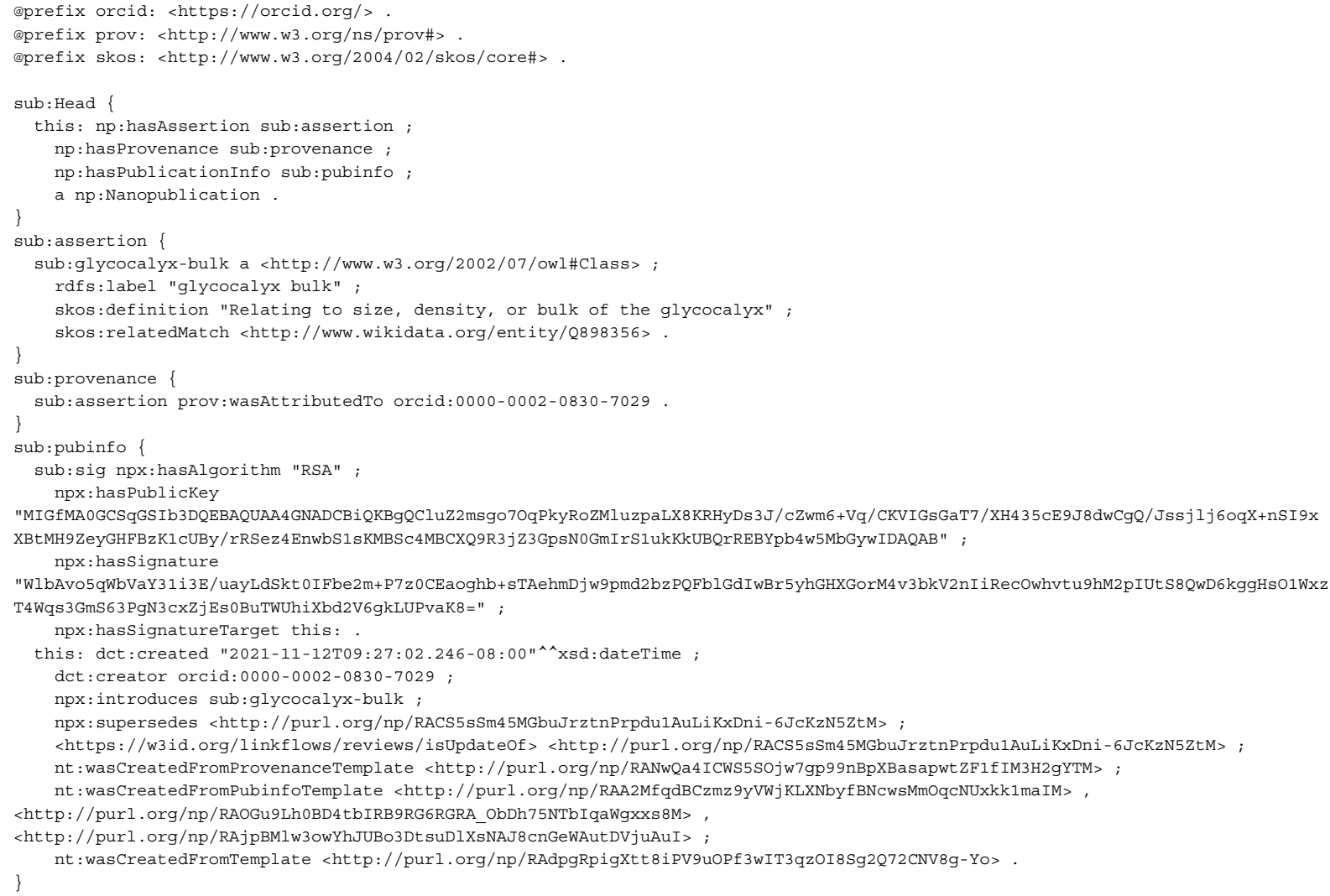

This is the class definition of "integrin clustering":

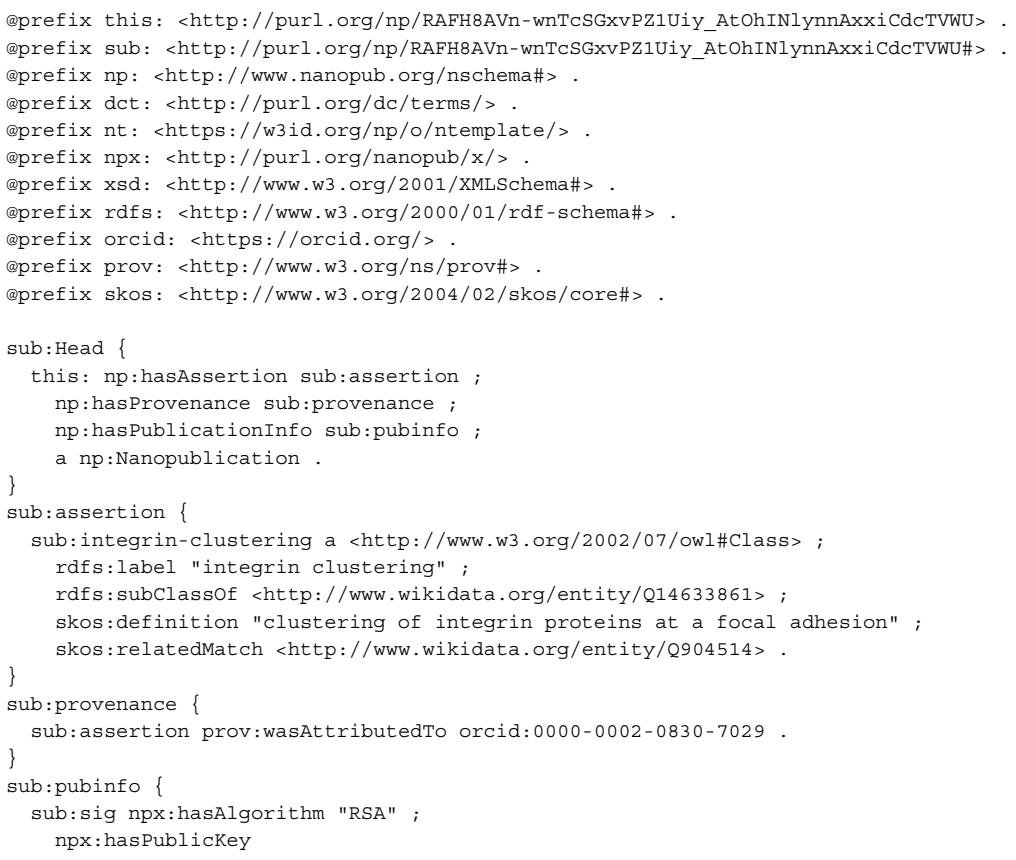




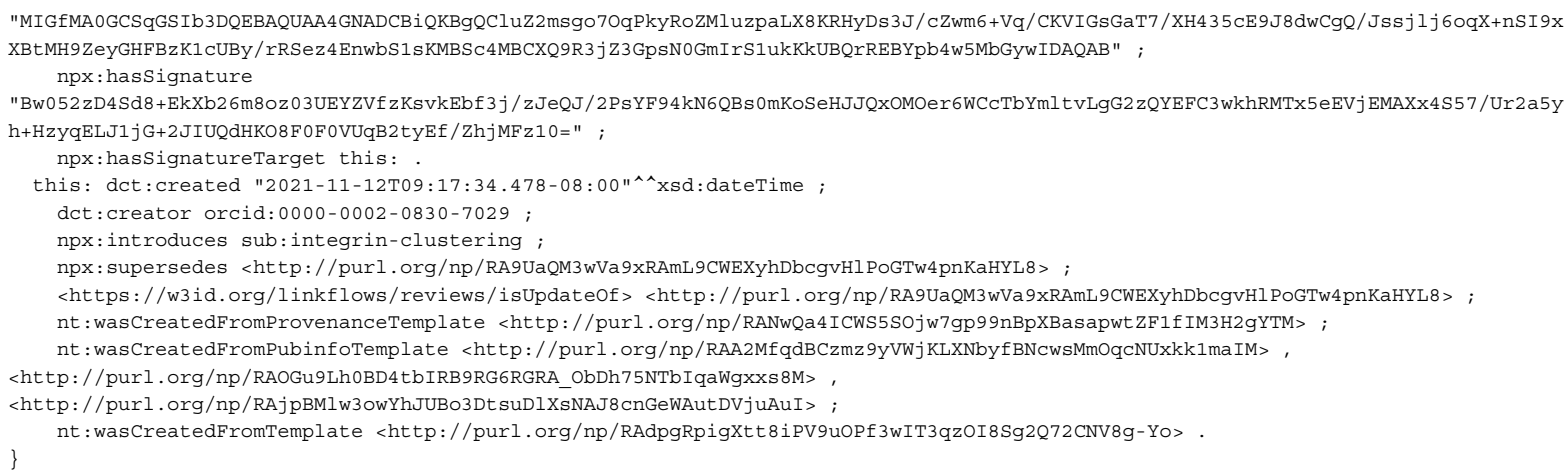

\section{References}

[1] C.I. Bucur, T. Kuhn, D. Ceolin and J. van Ossenbruggen, Expressing high-level scientific claims with formal semantics, in: Proceedings of the 11th Knowledge Capture Conference, 2021. doi:10.1145/3460210.3493561.

[2] M. Paszek, C. DuFort, O. Rossier et al., The cancer glycocalyx mechanically primes integrin-mediated growth and survival, Nature 511 (2014), 319-325. doi:10.1038/nature13535. 